\title{
PRAXISMANAGEMENT
}

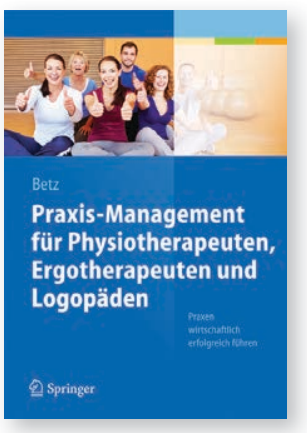

Mit Erfolg selbstständig sein > Ergotherapeuten befassen sich nicht gerne mit Betriebswirtschaft, sondern viel lieber mit medizinischen Fragen. Für Praxisinhaber sowie angehende Selbstständige sind jedoch Kenntnisse aus dem Bereich Management und Unternehmensführung essenziell. Nur so können sie den Erfolg ihrer Praxis sichern und Klienten langfristig behandeln. Die Kernaussage des Buchs könnte somit lauten: Erfolg ist kein Zufall, sondern das Produkt guter Planung. Das belegen auch relevante Beispiele. Bei der Auswahl der Marketinginstrumente helfen z. B. konkrete Tipps. Rechenbeispiele erleichtern Personalentscheidungen und konkrete Planungshilfen unterstützen die Kalkulation von Selbstzahlerangeboten.
Die Schwerpunkte der sechs Buchkapitel umfassen die Bereiche Unternehmensführung, Marketing, Personal-, Kosten- und Innovationsmanagement. Kästen, Merksätze und Grafiken veranschaulichen die Inhalte, nehmen auf einigen Seiten allerdings überhand, was das Buch etwas unübersichtlich macht. Eine farbige Gestaltung anstatt der schwarzweißen Darstellungen hätte hier sicherlich Abhilfe geschaffen.

Ein praxisnahes, verständliches Nachschlagewerk, in dem Praxisinhaber und angehende Selbstständige viele Anregungen und Antworten auf ihre Fragen erhalten.

Ina Koetz, Dipl.-Physiotherapeutin (FH) aus Osnabrück

\section{B. Betz}

Praxis-Management für Physiotherapeuten, Ergotherapeuten und Logopäden Springer Verlag 2014, 262 S., 34,99€ ISBN 978-3-642-38406-6

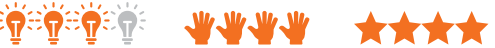

\section{DEMENZ}

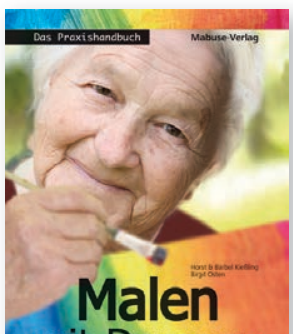

mit Demenz
Neue Impulse >

Dieses Praxishandbuch bietet Therapeuten, Pflegekräften, Angehörigen und Pädagogen einen Anreiz, kreativ mit Menschen mit Demenz zu arbeiten. Zwei Kunstpädagogen und eine Grafikdesignerin zeigen, dass die Zielgruppe aufgrund ihrer noch vorhandenen handwerklichen und kreativen Ressourcen durchaus Erfolge im Malen erzielen kann. Sie weisen aber auch darauf hin, dass dies nur in der ersten Phase der Krankheit möglich ist. Der Grundgedanke des Buchs ist, Menschen mit Demenz etwas zuzutrauen, Impulse zu geben und vor allem einen wertschätzenden und respektvollen Umgang mit ihnen zu fördern.

Das Buch beinhaltet 15 verschiedene und mit unterschiedlichen Arbeitsmaterialien erprobte Arbeitseinheiten. Diese sind nach Schwierigkeitsgraden gestaffelt, sodass die Therapeutin vorhandene Ressourcen ihrer
Klienten abtasten und eine Überforderung vermeiden kann. Der methodische Ablauf jeder Einheit ist Schritt für Schritt dargestellt: beginnend mit einem Gedicht, über die praktischen Übungen bis hin zur Verabschiedung. Die Materialaufstellungen zu jeder Übung zeigen, dass die Vorbereitungszeiten kurz sind. Im letzten Buchteil findet der Leser Tipps, um Ausstellungen zu arrangieren und Bilder zu rahmen, sowie weiterführende Literatur.

Ein tolles Buch! Es macht Lust, es auszuprobieren. Die Autoren führten das Malprogramm im Rahmen des Projekts „Demente Menschen in der Kommune“ immer einmal im Monat durch. Ich empfehle es allen, die Malkurse für diese Zielgruppe geben möchten.

Kristin Decker, Ergotherapeutin in einem Pflegeheim in Markkleeberg

H. Kießling, B. Kießling, B. Osten Malen mit Demenz

Mabuse Verlag 2014, 59 S., 19,90€

ISBN 978-3-86321-180-6

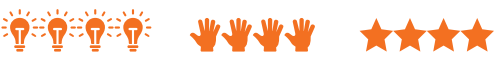

\section{LESEREMPFEHLUNG}

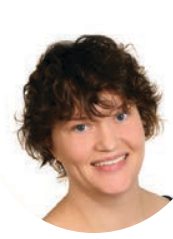

Authentisch und berührend > Daniela Schreiter, eine Asperger-Autistin, erzählt in ihrem autobiografischen Comic, wie es ist, mit dieser Diagnose zu leben. In intimen Anekdoten zeichnet sie die Schwierigkeiten von ihrer Kindheit bis zum Erwachsenenalter auf. Wie sie zum Beispiel mühsam lernen musste, über ihren Schatten zu springen, um so akzeptiert zu werden, wie sie ist. Warum ein normaler Smalltalk sie vor ungeahnte Hürden stellt und weshalb plötzliche, laute Geräusche Schmerzen verursachen können. Dieser Comic schafft es, autistische Wahrnehmung amüsant und faszinierend darzustellen - eine ungewöhnliche Alternative zur gängigen Literatur. Menschen mit Asperger können sich in dem Buch wiederfinden und erfahren, dass sie mit ihrem Anderssein nicht alleine

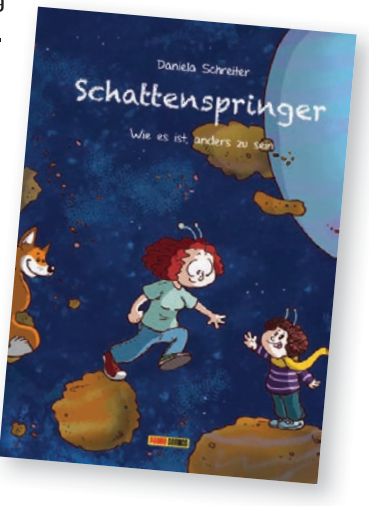
sind. Therapeuten, Eltern und Lehrer haben die Möglichkeit, die autistische Welt zu begreifen. Ein absolut empfehlenswertes Buch. Es klärt authentisch und berührend über ein sensibles Thema auf, ohne belehrend zu wirken.

Katarina Hildebrandt, Ergotherapeutin aus München

D. Schreiter

\section{Schattenspringer}

2. Auflage

Panini Verlag 2014, 160 S., 19,99€

ISBN 978-3-86201-950-2

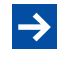

\section{LESEREMPFEHLUNG}

Wollen Sie Ihren Kolleginnen und Kollegen ein Buch empfehlen? Schicken Sie Ihren Vorschlag an: ergopraxis@thieme.de. Wir belohnen die besten Tipps mit einem Platz in ergopraxis und einem 20-Euro-Thieme-Buchgutschein! 